\title{
Crack Problem in Nested Thick Pipes
}

\author{
İç İçe Geçmiş Kalın Borularda Çatlak Problemi
}

\author{
Nurcan ASCI DEMIROZ ${ }^{* 1, a}$, Elif PUL ${ }^{2, b}$ \\ ${ }^{1}$ Inonu Street, Highways Lodgements, $A^{\text {th }}$ Block, Building No.114/7, 61040, Trabzon \\ ${ }^{2}$ Regional Offices of Provincial Bank, 61000, Trabzon
}

• Geliş tarihi / Received: 26.10.2018 • Düzeltilerek geliş tarihi / Received in revised form: 28.01.2019 • Kabul tarihi / Accepted: 04.02.2019

\begin{abstract}
The paper considers the elastostatic axisymmetric problem for long nested thick-walled cylinders with two different crack cases under uniform axial load according to the theory of elasticity. Both of cylinders have different elastic constants. Using the Fourier and Hankel transform techniques, the problem is formulated in terms of an integral equation which has a simple Cauchy kernel. The singular integral equation is solved numerically. Some results for the stress intensity factors are presented graphically.
\end{abstract}

Keywords: Crack, Integral transform technique, Singular integral equation, Stress-intensity factor

$\ddot{O} z$

Bu çalışmada düzgün yayılı çekmeye maruz iç içe geçmiş eksenel simetrik sonsuz uzun kalın borularda çatlak problemi elastisite teorisine göre incelenmiştir. Her iki borunun da elastik özellikleri birbirinden farklıdır. Fourier and Hankel dönüşüm tekniklerinden yararlanılarak problem basit Cauchy çekirdeğine sahip bir tekil integral denklem sistemine indirgenmiştir. Bu tekil integral sistemi, Gauss-Chebyshev integrasyon formülü kullanilarak saylsal olarak çözülmüş̧ür. Gerilme şiddeti faktörleri hesaplanmış ve grafik olarak sunulmuştur.

Anahtar Kelimeler: Çatlak, İntegral dönüşüm tekniği, Tekil integral, Gerilme yoğunluk faktörü

\footnotetext{
*a Nurcan ASCI DEMIROZ, nurcan_asci@ hotmail.com; Tel: (0532) 47605 75; orcid.org/0000-0002-8461-5138

${ }^{\mathrm{b}}$ orcid.org/0000-0001-8506-7906
} 


\section{Introduction}

The problems of a cylinder containing a crack are important for many engineering applications such as pressure vessels, pipes and other cylindrical containers. In this type of problems, axisymmetric field of displacements is often used due to its appropriateness. To find the maximum load to be carried by the structure with a crack, the most commonly used criteria is the stress intensity factor for linear elastic materials.

Many studies were done in the field of cylinder problems that contain cracks in the past. Sneddon and Welch (1963) examined the distribution of stress in a long and elastic cylinder containing a penny-shaped crack. Sneddon and Tait (1963) treated the effect of a penny shaped crack on distribution of stress in a long circular cylinder. Erdogan and Ratwani (1972) investigated the fracture problem for various geometric shaped shells with a through crack. Gupta (1974) investigated the problem of a semi-infinite cylinder with axial symmetry. Erdogan and Erdol (1978) examined an axisymmetric ring shaped internal and edge crack problem of hollow circular cylinder. The problem of a long cylinder containing a circumferential crack in axially symmetric under various loadings was solved by Nied and Erdogan (1983). The elastostatic axisymmetric problem for a long thick-walled cylinder containing an axisymmetric circumferential crack has been investigated by Birinci (2002), in which the crack was considered to be internal or edge crack with cladding. Altundag Artem and Gecit (2002) concerned with the fracture of an axisymmetric hollow cylindrical bar containing rigid inclusions. The cylinder is under the action of uniformly distributed axial tension applied at infinity. Toygar and Geçit (2006) considered the problem of an axisymmetric infinite cylinder with a ring shaped crack at $\mathrm{z}=0$ and two ring shaped rigid inclusions with negligible thickness at $z= \pm L$. The cylinder is under the action of uniformly distributed axial tension applied at infinity and its lateral surface is free of fraction. The elastostatic axisymmetric problem for a long thick walled cylinder containing internal crack with two cladding was investigated by Birinci et al. (2006). Aydin and Altundag Artem (2008) examined the fracture of an infinite thick-walled cylinder. The inner surface of the cylinder is stress free and the outer is rigidly fixed. The cylinder having a ring-shaped crack located at the symmetry plane is subjected to distributed compressive load on its surface.
In this paper, the stress intensity factors at the crack tips for nested thick pipes containing a ringshaped crack under axial symmetry are studied. In Fig. 1, two nested hollow-circular cylinders are shown. The cylinders are assumed to be elastic, homogeneous, and isotropic. The inner cylinder with inner radius ' $\mathrm{a}$ ' and outer radius ' $\mathrm{e}$ ' is included a crack having axial symmetry. The outer cylinder has inner radius ' $e$ ' and outer radius 'b'. The system is subjected to uniformly distributed tensile load. Two cases are considered: a) the internal crack case $(\mathrm{a}<\mathrm{c}<\mathrm{d}<\mathrm{e})$, and (b) the internal edge crack case $(\mathrm{a}=\mathrm{c}<\mathrm{d}<\mathrm{e})$. The elastic properties of the cylinders 1 and 2 are different. The effect of the body forces is neglected.

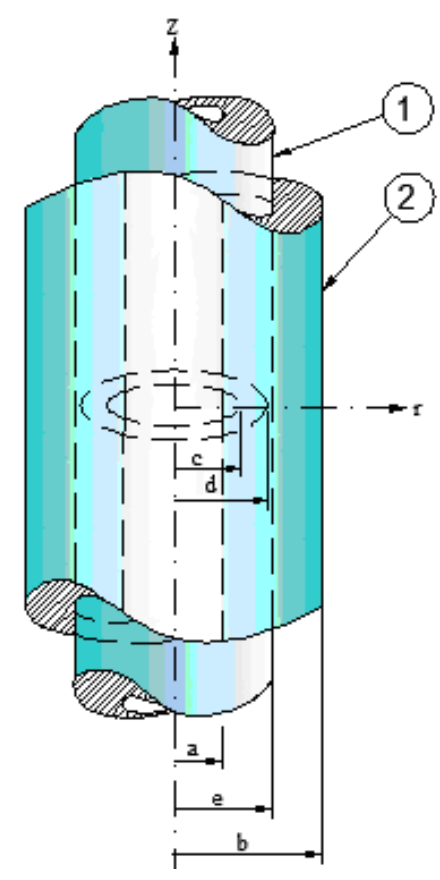

Fig. 1. Geometry of the problem

\section{Derivation of Integral Equation}

The solution of the problem in Fig. 1 is obtained by using the superposition of solutions for two different problems such that an axisymmetric infinite thick pipe problem subjected to only a uniform axial tensile loads with no crack, and an axisymmetric infinite thick pipe problem containing annular crack at $\mathrm{z}=0$ symmetry plane. Crack free surface can be obtained under the influence of the uniform tensile load $\mathrm{P}_{\mathrm{o}}$ with the superposition of these two cases (Gupta, 1974; Erdol and Erdogan, 1978; Artem and Gecit, 2002). The main problem in this paper is actually the second one, because the first part was solved in the earlier studies (Erdol and Erdogan, 1978; Gecit, 1986). For this purpose, the stresses $f(r)$ are 
obtained from the solution of the first problem, then they are applied to the crack surfaces in opposite direction as external load in the problem of axisymmetric hollow cylinder containing a crack. Because the $\mathrm{z}=0$ is symmetry plane, only the interval $0 \leq z<\infty$ is considered. Boundary conditions of the problem can be written as follows:

$$
\begin{array}{ll}
\sigma_{r r}^{1}(a, z)=0 & 0 \leq z \leq \infty \\
\tau_{r z}^{1}(a, z)=0 & 0 \leq z \leq \infty \\
\sigma_{r r}^{2}(b, z)=0 & 0 \leq z \leq \infty \\
\tau_{r z}^{2}(b, z)=0 & 0 \leq z \leq \infty \\
\tau_{r z}^{1}(r, 0)=\tau_{r z}^{2}(r, 0)=0 & a \leq r<b \\
U_{r}^{(1)}(e, z)=U_{r}^{(2)}(e, z) & \\
U_{z}^{(1)}(e, z)=U_{z}^{(2)}(e, z) & \\
\sigma_{r r}^{(1)}(e, z)=\sigma_{r r}^{(2)}(e, z) & \\
\tau_{r z}^{(1)}(e, z)=\tau_{r z}^{(2)}(e, z) & \\
\sigma_{z z}^{(1)}(r, 0)=-f(r) & c<r<d
\end{array}
$$

$U_{z}(r, 0)=0 \quad a<r<c, d<r<b$

$\lim _{z \rightarrow 0} \frac{\partial U_{z}}{\partial r}=0 \quad a<r<c, d<r<b$

$\lim _{z \rightarrow 0} \frac{\partial U_{r}}{\partial r}=G(r) \quad c<r<d$

Compatibility condition related with displacements through the $\mathrm{z}$ axis of points along the crack can be written as follows,

$\int_{c}^{d} \frac{\partial U_{z}}{\partial r} d r \quad c \leq r \leq d$

where $U_{r}, U_{z}$ are $r$ and $z$ components of the displacement vector, respectively.

The plane of the ring crack symmetry is taken as the plane where the $\mathrm{z}$ axis of the cylinder is zero. The solution of the axial symmetry infinite cylinder without crack obtained by using the Fourier and Hankel transforms is added to the solution of the half space loaded (Erdol and Erdogan, 1978), which also has axial symmetry characterizing the crack. Thus, the displacement and the relevant stress components of problem for cylinders 1 and 2 are obtained as follows, respectively.

$$
\begin{aligned}
& U_{r_{1}(r, z)}=-\frac{1+v_{1}}{E_{1}} \sqrt{\frac{2}{\pi}} \int_{0}^{\infty} \xi^{2}\left[A_{1}(\xi) I_{1}(\xi r)+B_{1}(\xi) \xi I_{0}(\xi r)-C_{1}(\xi) K_{1}(\xi r)-D_{1}(\xi) \xi r K_{0}(\xi r)\right] \operatorname{Cos}(\xi z) d \xi \\
& U_{z_{1}(r, z)}=\frac{1+v_{1}}{E_{1}}\left\langle\sqrt { \frac { 2 } { \pi } } \int _ { 0 } ^ { \infty } \xi ^ { 2 } \left\{ A_{1}(\xi) I_{0}(\xi r)+B_{1}(\xi)\left[4\left(1-v_{1}\right) I_{0}(\xi r)+\xi r I_{1}(\xi r)\right]+C_{1}(\xi) K_{0}(\xi r)+\right.\right. \\
& \left.\left.D_{1}(\xi)\left[\xi r K_{1}(\xi r)-4\left(1-v_{1}\right) K_{0}(\xi r)\right]\right\} \operatorname{Sin}(\xi z) d \xi+\int_{0}^{\infty} \lambda^{3}\left[\frac{\left(4 v_{1}-2-z \lambda\right) F(\lambda)}{\lambda}-E F(\lambda)\right] e^{-\lambda z} J_{0}(\lambda r) d \lambda\right\rangle
\end{aligned}
$$$$
\sigma_{r_{1}}(r, z)=\sqrt{\frac{2}{\pi}} \int_{0}^{\infty} \xi^{2}\left\{A_{1}(\xi)\left[-\xi I_{0}(\xi r)+I_{1}(\xi r) / r\right]+B_{1}(\xi) \xi\left[4\left(2 v_{1}-1\right) I_{0}(\xi r)-\xi r I_{1}(\xi r)\right]-\right.
$$$$
\left.C_{1}(\xi)\left[\xi K_{0}(\xi r)+K_{1}(\xi r) / r\right]+D_{1}(\xi) \xi\left[\left(1-2 v_{1}\right) K_{0}(\xi r)-\xi r K_{1}(\xi r)\right]\right\} \operatorname{Cos}(\xi z) d \xi+\int_{0}^{\infty} \lambda^{3}\left\{E \lambda \left[\frac{J_{1}(\lambda r)}{r}-\right.\right.
$$$$
\left.\left.\lambda J_{0}(\lambda r)\right]+F(\lambda)\left[\left(2 v_{1}-\lambda z+1\right) J_{0}(\lambda r)+(\lambda z-1) J_{1}(\lambda r) / \lambda r\right]\right\} e^{-\lambda z} d \lambda
$$

$\sigma_{\theta_{1}}(r, z)=$

$\sqrt{\frac{2}{\pi}} \int_{0}^{\infty} \xi^{3}\left\{\frac{A_{1}(\xi) I_{1}(\xi r)}{r}+B_{1}(\xi) \xi\left(2 v_{1}-1\right) I_{0}(\xi r)+C_{1}(\xi) K_{1}(\xi r) / \xi r+D_{1}(\xi)\left(1-2 v_{1}\right) K_{0}(\xi r)\right\} \operatorname{Cos}(\xi z) d \xi+$

$\int_{0}^{\infty} \lambda^{3}\left\{-E(\lambda) \frac{J_{1}(\lambda r)}{r}+F(\lambda)\left[2 v_{1} J_{0}(\lambda r)+(1-\lambda z) J_{1}(\lambda r) / \lambda r\right]\right\} e^{-\lambda z} d \lambda$

$$
\begin{aligned}
& \sigma_{z_{1}}(r, z)= \\
& \sqrt{\frac{2}{\pi}} \int_{0}^{\infty} \xi^{3}\left\{A_{1}(\xi) I_{0}(\xi r)+B_{1}(\xi)\left[2\left(2 v_{1}-1\right) I_{0}(\xi r)+\xi r I_{1}(\xi r)\right]+C_{1}(\xi) K_{0}(\xi r)+D_{1}(\xi)\left[\xi r K_{1}(\xi r)-\right.\right. \\
& \left.\left.2\left(2-v_{1}\right) K_{0}(\xi r)\right]\right\} \operatorname{Cos}(\xi z) d \xi+\int_{0}^{\infty} \lambda^{3}\left[\lambda E(\lambda)+F(\lambda)\left(\lambda z+1-2 v_{1}\right)\right] e^{-\lambda z} J_{0}(\lambda r) d \lambda
\end{aligned}
$$


$\tau_{r z_{1}}=\sqrt{\frac{2}{\pi}} \int_{0}^{\infty} \xi^{3}\left\{A_{1}(\xi) I_{1}(\xi r)+B_{1}(\xi)\left[2\left(1-v_{1}\right) I_{1}(\xi r)+\xi r I_{0}(\xi r)\right]-C_{1}(\xi) K_{1}(\xi r)-D_{1}(\xi)\left[\xi r K_{0}(\xi r)-\right.\right.$

$\left.\left.2\left(1-v_{1}\right) K_{1}(\xi r)\right]\right\} \operatorname{Sin}(\xi z) d \xi+\int_{0}^{\infty} \lambda^{3}\left[\lambda E(\lambda)+F(\lambda)\left(\lambda z-2 v_{1}\right)\right] e^{-\lambda z} J_{1}(\lambda r) d \lambda$

$U_{r_{2}}(r, z)=$

$-\frac{1+v_{2}}{E_{2}} \sqrt{\frac{2}{\pi}} \int_{0}^{\infty} \xi^{2}\left[A_{2}(\xi) I_{1}(\xi r)+B_{2}(\xi) \xi r I_{0}(\xi r)-C_{2}(\xi) K_{1}(\xi r)-D_{2}(\xi) \xi r K_{1}(\xi r)\right] \operatorname{Cos}(\xi z) d \xi$

$U_{z_{2}}(r, z)=\frac{1+v_{2}}{E_{2}} \sqrt{\frac{2}{\pi}} \int_{0}^{\infty} \xi^{2}\left\langle A_{2}(\xi) I_{1}(\xi r)+B_{2}(\xi)\left[4\left(1-v_{2}\right) I_{0}(\xi r)+\xi r I_{1}(\xi r)\right]+C_{2}(\xi) K_{0}(\xi r)+\right.$

$\left.D_{2}(\xi)\left[\xi r K_{1}(\xi r)-4\left(1-v_{2}\right) K_{0}(\xi r)\right]\right\rangle \operatorname{Sin}\left(\xi_{Z}\right) d \xi$

$\sigma_{r_{2}}(r, z)=$

$\sqrt{\frac{2}{\pi}} \int_{0}^{\infty} \xi^{2}\left\{A_{2}(\xi)\left[-\xi I_{0}(\xi r)+I_{1}(\xi r) / r\right]+B_{2}(\xi) \xi\left[\left(2 v_{2}-1\right) I_{0}(\xi r)-\xi r I_{1}(\xi r)\right]-C_{2}(\xi)\left[\xi K_{0}(\xi r)+\right.\right.$

$\left.\left.\frac{K_{1}(\xi r)}{r}\right]+D_{2}(\xi) \xi\left[\left(1-2 v_{2}\right) K_{0}(\xi r)-\xi r K_{1}(\xi r)\right]\right\} \operatorname{Cos}(\xi z) d \xi$

$\sigma_{\theta_{2}}(r, z)=\sqrt{\frac{2}{\pi}} \int_{0}^{\infty} \xi^{3}\left\{-\frac{A_{2}(\xi) I_{1}(\xi r)}{\xi r}+B_{2}(\xi) \xi\left(2 v_{2}-1\right) I_{0}(\xi r)+C_{2}(\xi) K_{1}(\xi r) / \xi r+D_{2}(\xi)(1-\right.$

$\left.\left.2 v_{2}\right) K_{0}(\xi r)\right\} \operatorname{Cos}(\xi z) d \xi$

$\sigma_{z_{2}}(r, z)=$

$\sqrt{\frac{2}{\pi}} \int_{0}^{\infty} \xi^{3}\left\{A_{2}(\xi) I_{0}(\xi r)+B_{2}(\xi)\left[2\left(2-v_{2}\right) I_{0}(\xi r)+\xi r I_{1}(\xi r)\right]+C_{2}(\xi) K_{0}(\xi r)+D_{2}(\xi)\left[\xi r K_{1}(\xi r)-\right.\right.$

$\left.\left.2\left(2-v_{2}\right) K_{0}(\xi r)\right]\right\} \operatorname{Cos}(\xi z) d \xi$

$\tau_{r z_{2}}=\sqrt{\frac{2}{\pi}} \int_{0}^{\infty} \xi^{3}\left\{A_{2}(\xi) I_{1}(\xi r)+B_{2}(\xi)\left[2\left(1-v_{2}\right) I_{1}(\xi r)+\xi r I_{0}(\xi r)\right]-C_{2}(\xi) K_{1}(\xi r)+D_{2}(\xi)\left[-\xi r K_{0}(\xi r)+\right.\right.$

$\left.\left.2\left(1-v_{2}\right) K_{1}(\xi r)\right]\right\} \operatorname{Sin}(\xi z) d \xi$

In the expressions given above, $I_{i}, K_{i}$ and $J_{i}(i=0,1)$ are the modified Bessel functions of the first and second kind, and the ith-order Bessel functions of the first kind, respectively. $A_{i}(\xi), B_{i}(\xi), C_{i}(\xi), D_{i}(\xi)$, $E(\lambda)$ and $F(\lambda)(i=1,2)$ are unknown coefficients, and they are calculated from the boundary conditions depending on $G(r)$. By using boundary condition in Eq. (5), Eq. (27) is obtained.

$\int_{0}^{\infty} \lambda^{3}\left[\lambda E(\lambda)-2 v_{1} F(\lambda)\right] J_{1}(\lambda r) d \lambda$

Assuming the term in parenthesis is equal to zero in Eq. (27), boundary condition Eq. (5) is constantly verified such that

$\lambda E(\lambda)-2 v_{1} F(\lambda)=0$

From Eq. (13), Eq. (29) can be written following form:

$\frac{\partial U_{Z 1}(r, 0)}{\partial r}=G(r)=\frac{2\left(1-v_{1}^{2}\right)}{E_{1}} \int_{0}^{\infty} \lambda^{4} F(\lambda) J_{1}(\lambda r) d \lambda$

where the function $G(r)$ is unknown. Applying the inverse Henkel transform of both sides in Eq. (29), Eq. (30) can be expressed as:

$\lambda^{3} F(\lambda)=\frac{E_{1}}{2\left(1-v_{1}^{2}\right)} \int_{c}^{d} \rho G(\rho) J_{1}(\lambda \rho) d \rho$ 
Making use of Eqs. (15)-(26) and Eqs. (28)-(29), the boundary conditions Eqs. (1)-(5) and Eqs. (8)-(10) may be written as:

$$
\begin{aligned}
& A_{1}(\xi)\left[-\xi a I_{1}(\xi a)+I_{1}(\xi a)\right]+\xi a B_{1}(\xi)\left[\left(2 v_{1}-1\right) I_{0}(\xi a)-\xi a I_{1}(\xi a)\right]-C_{1}(\xi)\left[\xi a K_{0}(\xi a)+K_{1}(\xi a)\right]+ \\
& \xi a D_{1}(\xi)\left[\left(1-2 v_{1}\right) K_{0}(\xi a)-\xi a K_{1}(\xi a)\right]=-\frac{1}{\xi^{2}} \frac{E_{1}}{2\left(1-v_{1}\right)^{2}} \sqrt{\frac{2}{\pi}} \int_{c}^{d} \rho\left\{\xi^{2} \rho a I_{0}(\xi a) K_{0}(\xi \rho)+\left[2\left(v_{1}-1\right)-\right.\right. \\
& \left.\left.(\xi a)^{2}\right] I_{1}(\xi a) K_{1}(\xi \rho)+\xi a I_{0}(\xi a) K_{1}(\xi \rho)-\xi \rho I_{1}(\xi a) K_{0}(\xi \rho)\right\} G(\rho) d(\rho) \\
& A_{1}(\xi) I_{1}(\xi a)+B_{1}(\xi)\left[2\left(1-v_{1}\right) I_{1}(\xi a)+\xi a I_{0}(\xi a)\right]-C_{1}(\xi) K_{1}(\xi a)+D_{1}(\xi)\left[2\left(1-v_{1}\right) K_{1}(\xi a)\right]= \\
& -\frac{1}{\xi^{2}} \frac{E_{1}}{2\left(1-v_{1}\right)^{2}} \sqrt{\frac{2}{\pi}} \int_{c}^{d} \rho\left\{\xi a I_{0}(\xi a) K_{1}(\xi \rho)-\xi \rho I_{1}(\xi a) K_{0}(\xi \rho)\right\} G(\rho) d(\rho)
\end{aligned}
$$

$$
\begin{aligned}
& A_{2}(\xi)\left[-\xi b I_{0}(\xi b)+I_{1}(\xi b)\right]+\xi b B_{2}(\xi)\left[\left(2 v_{2}-1\right) I_{0}(\xi b)-\xi b I_{1}(\xi b)\right]-C_{2}(\xi)\left[\xi b K_{0}(\xi b)+K_{1}(\xi b)\right]+ \\
& \xi b D_{2}(\xi)\left[\left(1-2 v_{2}\right) K_{0}(\xi b)-\xi b K_{1}(\xi b)\right]=0
\end{aligned}
$$

$A_{2}(\xi) I_{1}(\xi b)+B_{2}(\xi)\left[2\left(1-v_{2}\right) I_{1}(\xi b)+\xi b I_{0}(\xi b)\right]-C_{2}(\xi) K_{1}(\xi b)+D_{2}(\xi)\left[2\left(1-2 v_{2}\right) K_{1}(\xi b)-\right.$ $\left.\xi b K_{0}(\xi b)\right]=0$

$-\left(\frac{1+v_{1}}{1+v_{2}}\right) \frac{E_{2}}{E_{1}}\left\{A_{1}(\xi) I_{1}(\xi e)+B_{1}(\xi) \xi e I_{0}(\xi e)-C_{1}(\xi) K_{1}(\xi e)-D_{1}(\xi) \xi e K_{0}(\xi e)\right\}+\left\{A_{2}(\xi) I_{1}(\xi e)+\right.$ $\left.B_{2}(\xi) \xi e I_{0}(\xi e)-C_{2}(\xi) K_{1}(\xi e)-D_{2}(\xi) \xi e K_{0}(\xi e)\right\}=-\frac{1}{\xi^{2}} \frac{E_{2}}{\left(1-v_{1}\right)\left(1+v_{2}\right)} \sqrt{\frac{2}{\pi}} \int_{c}^{d} \rho\left\{2\left(1-v_{1}\right) I_{1}(\xi \rho) K_{1}(\xi e)+\right.$ $\left.\xi e I_{1}(\xi \rho) K_{0}(\xi e)-\xi \rho K_{1}(\xi e) I_{0}(\xi \rho)\right\} G(\rho) d(\rho)$

$-\left(\frac{1+v_{1}}{1+v_{2}}\right) \frac{E_{2}}{E_{1}}\left\{A_{1}(\xi) I_{1}(\xi e)+B_{1}(\xi)\left[4\left(1-v_{1}\right) I_{0}(\xi e)+\xi e I_{1}(\xi e)\right]+C_{1}(\xi) K_{0}(\xi e)+D_{1}(\xi)\left[\xi e K_{1}(\xi e)-\right.\right.$ $\left.\left.4\left(1-v_{1}\right) K_{0}(\xi e)\right]\right\}-\left\{A_{2}(\xi) I_{0}(\xi e)+B_{2}(\xi) \xi\left[4\left(1-v_{2}\right) I_{0}(\xi e)+\xi e I_{1}(\xi e)\right]+C_{2}(\xi) K_{0}(\xi e)+\right.$ $\left.D_{2}(\xi)\left[-4\left(1-v_{2}\right) K_{0}(\xi e) \xi e+K_{1}(\xi e)\right]\right\}=-\frac{1}{\xi^{2}} \frac{E_{2}}{\left(1-v_{1}\right)\left(1+v_{2}\right)} \sqrt{\frac{2}{\pi}} \int_{c}^{d} \rho\left\{\xi e I_{1}(\xi \rho) K_{1}(\xi e)-2(1-\right.$ $\left.\left.v_{1}\right) I_{1}(\xi \rho) K_{0}(\xi e)-\xi \rho K_{0}(\xi e) I_{0}\right\} G(\rho) d(\rho)$

$A_{1}(\xi)\left[-\xi I_{0}(\xi e)+\frac{I_{0}(\xi e)}{e}\right]+\xi B_{1}(\xi)\left[\left(2 v_{1}-1\right) I_{0}(\xi e)+\xi e I_{1}(\xi e)\right]-C_{1}(\xi)\left[-\xi K_{0}(\xi e)+\frac{K_{1}(\xi e)}{e}\right]+$ $\xi D_{1}(\xi)\left[\left(1-2 v_{1}\right) K_{0}(\xi e)-\xi e K_{1}(\xi e)\right]-A_{2}(\xi)\left[-\xi I_{0}(\xi e)+\frac{I_{1}(\xi e)}{e}\right]-\xi B_{2}(\xi)\left[\left(2 v_{2}-1\right) I_{0}(\xi e)-\right.$ $\left.\xi e I_{1}(\xi e)\right]-C_{2}(\xi)\left[\xi K_{0}(\xi e)+\frac{K_{1}(\xi e)}{e}\right]-\xi D_{2}(\xi)\left[\left(1-2 v_{2}\right) K_{0}(\xi e)-\xi e K_{1}(\xi e)\right]=$ $-\frac{1}{\xi^{2}} \frac{E_{1}}{2\left(1-v_{1}^{2}\right)} \sqrt{\frac{2}{\pi}} \int_{c}^{d} \rho\left\{\left[2\left(v_{1}-1\right)-(\xi e)^{2}\right] I_{1}(\xi \rho) K_{1}(\xi e)-\xi e I_{1}(\xi \rho) K_{0}(\xi e)+(\xi \rho) K_{1}(\xi e) I_{0}(\xi \rho)+\right.$ $\left.\xi^{2} \rho e K_{0}(\xi e) I_{0}(\xi \rho)\right\} G(\rho) d(\rho)$

$A_{1}(\xi) I_{1}(\xi e)+B_{1}(\xi)\left[2\left(1-v_{1}\right) I_{1}(\xi e)+\xi e I_{0}(\xi e)\right]-C_{1}(\xi) K_{1}(\xi e)+D_{1}(\xi)\left[2\left(1-v_{1}\right) K_{1}(\xi e)\right]+$ $D_{1}(\xi)\left[-\xi e K_{0}(\xi e)\right]-A_{2}(\xi) I_{1}(\xi e)-B_{2}(\xi)\left[2\left(1-v_{2}\right) I_{1}(\xi e)+\xi e I_{0}(\xi e)\right]+C_{2}(\xi) K_{2}(\xi e)-D_{2}(\xi)[2(1-$ $\left.\left.v_{2}\right) K_{2}(\xi e)-\xi e K_{0}(\xi e)\right]=-\frac{1}{\xi^{2}} \frac{E_{1}}{2\left(1-v_{1}^{2}\right)} \sqrt{\frac{2}{\pi}} \int_{c}^{d} \rho\left\{\xi \rho K_{1}(\xi e) I_{0}(\xi \rho)-\xi \rho e K_{0}(\xi e) I_{1}(\xi \rho)\right\} G(\rho) d(\rho)$

Now, substituting $A_{i}(\xi), B_{i}(\xi), C_{i}(\xi), D_{i}(\xi), E(\lambda)$ and $F(\lambda)$ given by Eqs. (31)-(38) into the condition Eq. (10), one may obtain the following singular integral equation for $G(\rho)$ :

$\frac{1}{\pi} \int_{c}^{d} G(\rho)\left\{\frac{1}{\rho-r}+K(\rho, r)\right\} d \rho=-\frac{2\left(1-v_{1}^{2}\right)}{E_{1}} f(r) \quad c<r<d$

The kernel $K(\rho, r)$ in Eq. (39) is

$K(\rho, r)=\frac{M(\rho, r)-1}{\rho-r}+\frac{M(\rho, r)}{\rho-r}-2 \rho L(\rho, r)$ 
$M(\rho, r)=\left\{\begin{array}{cl}E\left(\frac{r}{\rho}\right) & \rho>r \\ \frac{r}{\rho} E\left(\frac{\rho}{r}\right)+\frac{p^{2}-r^{2}}{\rho r} K\left(\frac{\rho}{r}\right) & \rho<r\end{array}\right.$

$L(\rho, r)=\int_{0}^{\infty} \frac{\xi}{\Delta}\left\{\left(\sum_{i=1}^{6} z_{i} a_{i}\right) I_{0}(\xi r)+\left(\sum_{i=1}^{6} z_{i} b_{i}\right)\left[2\left(2-v_{1}\right) I_{0}(\xi r)+\xi r I_{1}(\xi r)\right]+\left(\sum_{i=1}^{6} z_{i} c_{i}\right) K_{0}(\xi r)+\right.$

$\left.\left(\sum_{i=1}^{6} z_{i} d_{i}\right)\left[-2\left(2-v_{1}\right) K_{0}(\xi r)+\xi r K_{1}(\xi r)\right]\right\} d \xi$

$\mathrm{K}$ and $\mathrm{E}$ are the complete elliptic integrals of the first and second kind in Eq. (41), respectively. $\Delta, a_{i}, b_{i}, c_{i}, d_{i}, z_{i}(i=1, \ldots, 6)$ in Eq. (42) are described in Appendix A. The singular integral equation given by Eq. (39) has a simple Cauchy type singularity. From Eq. (14), Eq. (39) must be solved under the following single-valuedness condition

$\int_{c}^{d} G(\rho) d \rho=0$.

\section{Solution of the Integral Equation}

\subsection{Internal Crack Case}

Referring to Fig. 1, if the crack is not opened, i.e., $a<c$ and $d<e$, the kernel function is always finite. In this case, integral equation is solved by certain numerical methods (Erdol and Erdogan, 1978; Erdogan and Gupta, 1972). For this purpose, the normalized quantities are defined in Eq. (44).

$\rho=\frac{d-c}{2} \tau+\frac{d+c}{2}, r=\frac{d-c}{2} z+\frac{d+c}{2}$

The singular integral equation in Eq. (39) and the single-valuedness condition Eq. (43) become as

$\frac{1}{\pi} \int_{-1}^{1} \phi(\tau)\left\{\frac{1}{\tau-z}+K(\tau, z)\right\} d \tau=$

$-\frac{2\left(1-v_{1}^{2}\right)}{E_{1}} f(z) \quad(-1<\tau<1)$

$\frac{1}{\pi} \int_{-1}^{1} \phi(\tau) d \tau=0$

where

$K(\tau, z)=\frac{d-c}{2} K(\rho, r)$

In this case, the index of the integral equation is $(+1)$, thus, the solution may be expressed as (Erdogan and Gupta, 1972),

$\phi(\tau)=G(\tau)\left(1-r^{2}\right)^{-\frac{1}{2}} \quad(-1<\tau<1)$

$G(\tau)$ in Eq. (48) is bounded in the closed interval $(-1 \leq \tau \leq 1)$. Then, using Gauss-Chebyshev integration equality (Erdogan and Gupta, 1972), Eqs. (45)-(46) are replaced by:

$$
\begin{aligned}
& \sum_{i=1}^{n} \frac{1}{n}\left\{\frac{1}{\tau_{i}-z_{k}}+K\left(\tau_{i}-z_{k}\right)\right\} G\left(\tau_{i}\right)= \\
& -\frac{2\left(1-v_{1}^{2}\right)}{E_{i}} f\left(z_{k}\right) \quad(k=1, \ldots, n-1) \\
& \sum_{i=1}^{n} \frac{\pi}{n} G\left(\tau_{i}\right)=0
\end{aligned}
$$

where

$$
\begin{aligned}
\tau_{i} & =\operatorname{Cos}\left(\frac{2 i-1}{2 n} \pi\right) & i & =1, \ldots, n \\
z_{k} & =\operatorname{Cos} \frac{k}{n} \pi & k & =1, \ldots, n-1
\end{aligned}
$$

Here, Eqs. (49) - (50) are solved. Then, stressintensity factors are calculated by

$$
\begin{aligned}
& k_{c}=\lim _{r \rightarrow c} \sqrt{2(c-r)} \sigma_{z_{1}}(r, 0)= \\
& \frac{E_{1}}{2\left(1-v_{1}^{2}\right)} \sqrt{\frac{d-c}{2}} G(-1) \\
& k_{d}=\lim _{r \rightarrow d} \sqrt{2(r-d)} \sigma_{z_{1}}(r, 0)= \\
& -\frac{E_{1}}{2\left(1-v_{1}^{2}\right)} \sqrt{\frac{d-c}{2}} G(+1)
\end{aligned}
$$

By using the interpolation formulas (Krenk, 1975), $G(-1)$ and $G(+1)$ are obtained from $G\left(\tau_{i}\right)(i=1, \ldots, N)$. These stress intensity factors are normalized as $K_{d}=k_{d} /\left(\sigma_{1} \sqrt{(d-c) / 2}\right)$ and $K_{c}=k_{c} /\left(\sigma_{1} \sqrt{(d-c) / 2}\right)$.

\subsection{Internal Edge Crack Case}

In this specific case, the kernel $L(\rho, r)$ has also point singularities at $\rho=a$ and $r=a$. Singular terms in the kernel of integral equation may be obtained as following form: 
$K_{s}(\rho, r)=\int_{0}^{\infty} \frac{1}{2 \sqrt{\rho r}} e-\xi(\rho+r-2 a)\left\{2 \xi^{2}(\rho-a)(r-\right.$

a) $+\xi[-3(\rho-a)-(r-a)+2]\} d \xi$

If the singular terms are replaced by their closedform integrals, the singularities in the kernels are removed. The closed-form integral of the above expression is:

$$
\begin{aligned}
& K_{k}(\rho, r)=\frac{1}{2 \sqrt{\rho r}}\left\{-\frac{1}{\rho+r-2 a}+\frac{6(r-a)}{(\rho+r-2 a)^{2}}-\right. \\
& \left.\frac{4(r-a)^{2}}{(\rho+r-2 a)^{3}}\right\}
\end{aligned}
$$

The kernel $K_{1}(\rho, r)$ may be designed as the total of three pieces as

$K_{1}(\rho, r)=K_{1}(\rho, r)-K_{s}(\rho, r)+K_{k}(\rho, r)$

Defining normalized variables in the following,

$\rho=(d-a) \tau+a \quad 0<\tau<1$

$r=(d-a) z+a \quad 0<z<1$

the integral Eq. (31) becomes

$\frac{1}{\pi} \int_{0}^{1}\left[\frac{1}{\tau-z}+(d-a) K_{1}(\tau, z)\right] \phi(\tau) d \tau=$

$-\frac{2\left(1-v_{1}^{2}\right)}{E_{1}} \sigma_{1} \quad 0<\tau<1$

where,

$\phi(\tau)=g(\tau)(1-\tau)^{-\frac{1}{2}}$

Using the technique described by Gupta and Erdogan (1974) and using the Gauss-Chebyshev integration formula (Erdogan and Gupta 1972), Eq. (60) is re-arranged in the form

$\sum_{i=1}^{n} \frac{1}{2 n+1}\left[\frac{1}{\tau_{i}-z_{k}}+(d-a) K_{1}\left(\tau_{i}, z_{k}\right)\right] g\left(\tau_{i}\right)=$

$-\frac{2\left(1-\sigma_{1}^{2}\right)}{E_{1}} \sigma_{1} \quad(k=1, \ldots, n)$

where,

$\tau_{i}=\operatorname{Cos}\left(\frac{2 i-1}{4 n+2} \pi\right) \quad(i=1, \ldots, n)$

$z_{k}=\operatorname{Cos}\left(\frac{k}{2 n+1} \pi\right) \quad(k=1, \ldots, n)$

The stress-intensity factor for the internal edge crack case may be given by $k_{d}=\lim _{\rho \rightarrow d} \sqrt{2(\rho-d)} \sigma_{z_{1}}(r, 0)=$

$-\frac{E_{1}}{2\left(1-v_{1}^{2}\right)} \sqrt{(d-a)} g(1)$

where $g(1)$ is defined Gupta and Erdogan (1974) by

$$
\begin{aligned}
& g(1)=\frac{2}{2 n+1} \sum_{j=1}^{n} \operatorname{Cot}\left[\frac{2 j-1}{2(2 n+1)} \pi\right] \operatorname{Sin}[(2 j- \\
& \text { 1) } \left.\frac{n}{2 n+1} \pi\right] g\left(\tau_{j}\right)
\end{aligned}
$$

The stress intensity factor $\left(k_{d}\right)$ is given in the normalized form as $K_{d}=k_{d} /\left(\sigma_{1} \sqrt{d-a}\right)$.

\section{Results and Discussion}

The numerical solutions are obtained for a uniform axial tension load applied at $\mathrm{z}=0$ plane of symmetry. Stress intensity factors at crack tips depending on the ratio of elastic constants and various geometric dimensionless quantities are obtained. The effect of geometric parameters on the stress-intensity factors has been investigated for two different crack cases. The results are presented in Tables 1-4 and Figs. 2-8.

Figs. 2 to 5 show the variation of the normalized stress-intensity factors with increasing crack length $(d / e)$ for the internal crack case. When the crack lengths are getting smaller, the values of $K_{c}$ and $K_{d}$ approach the unity. This is expected since the problem reduces to that of a finite crack in an infinite medium when the ratio $\{[(d-c) / e]=$ $\left.1 \times 10^{-6}\right\}$ is small enough.

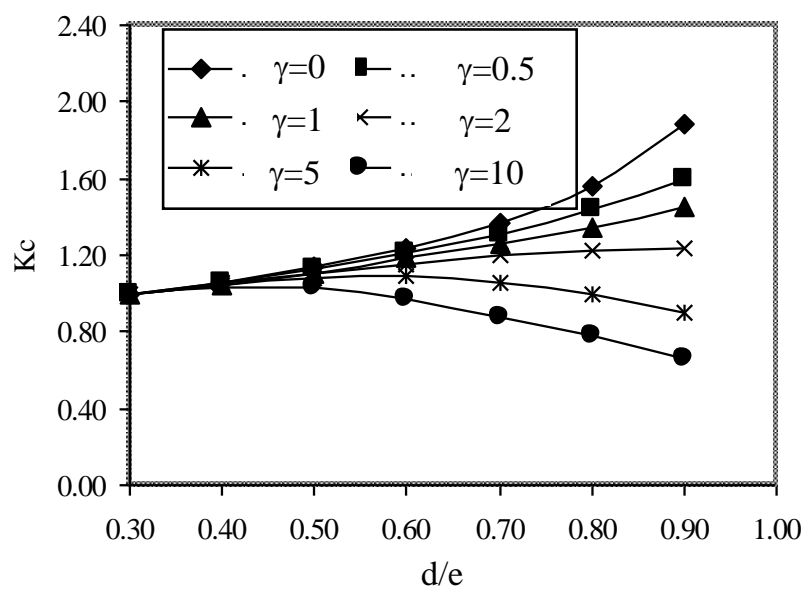

Fig. 2. Variation of normalized $\mathrm{K}_{\mathrm{c}}$ with crack length (d/e) for an internal crack

$$
\begin{aligned}
(a / e=0.1, c / e= & 0.2, b / e=1.5, \gamma \\
& =E_{2}\left(1+v_{1}\right) / E_{1}\left(1+v_{2}\right), K_{c} \\
& \left.=k_{c} /\left(P_{0} \sqrt{(d-c) / 2}\right)\right)
\end{aligned}
$$


It may be seen in Tables 1 and 2 and Figs. 2 to 6 that $K_{c}$ is always bigger than $K_{d}$. In case the stiffness of the inner pipe is greater than that of the outer one $(\gamma \leq 1)$, the values of the stress intensity factors at the crack tips also increase with increasing crack width. On the other hand, in case the stiffness of the inner pipe is smaller than that of the outer one $(\gamma>1)$, the values of the stress intensity factors at the crack tips gradually decrease with increasing the crack length as seen in Figs. 2 and 3.

Another observation is that for a certain rate of shear moduli, the values of the stress intensity factors at the crack tips increase with decreasing of the thickness of the inner and outer pipes both. The values of the stress intensity factors decrease at the crack tips for the internal crack case and approach a constant asymptotic value as shown in Fig. 6. These results are also presented in Tables 1 and 2 for various $\gamma$ and (b/e) values.

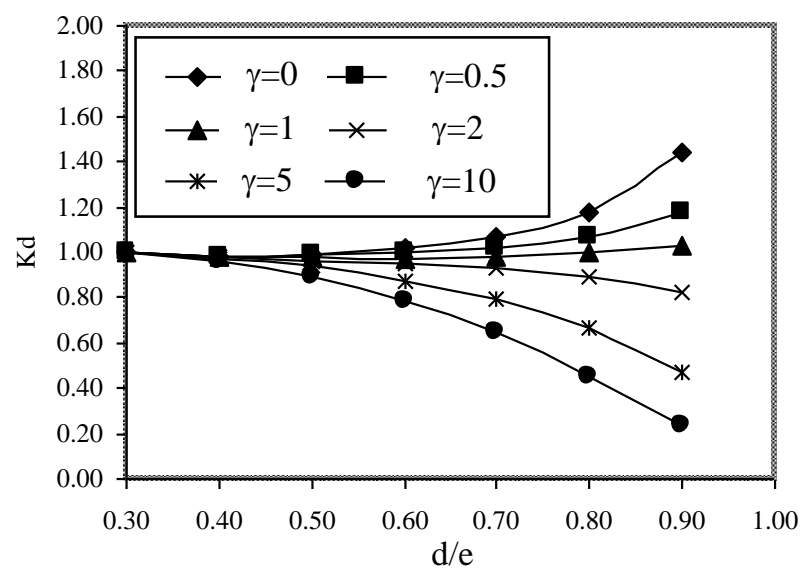

Fig. 3. Variation of normalized $\mathrm{K}_{\mathrm{d}}$ with crack length $(\mathrm{d} / \mathrm{e})$ for an internal crack

$$
\begin{aligned}
(a / e=0.1, c / e & =0.2, b / e=1.5, \gamma \\
& =E_{2}\left(1+v_{1}\right) / E_{1}\left(1+v_{2}\right), K_{d} \\
& \left.=k_{d} /\left(P_{0} \sqrt{(d-c) / 2}\right)\right)
\end{aligned}
$$

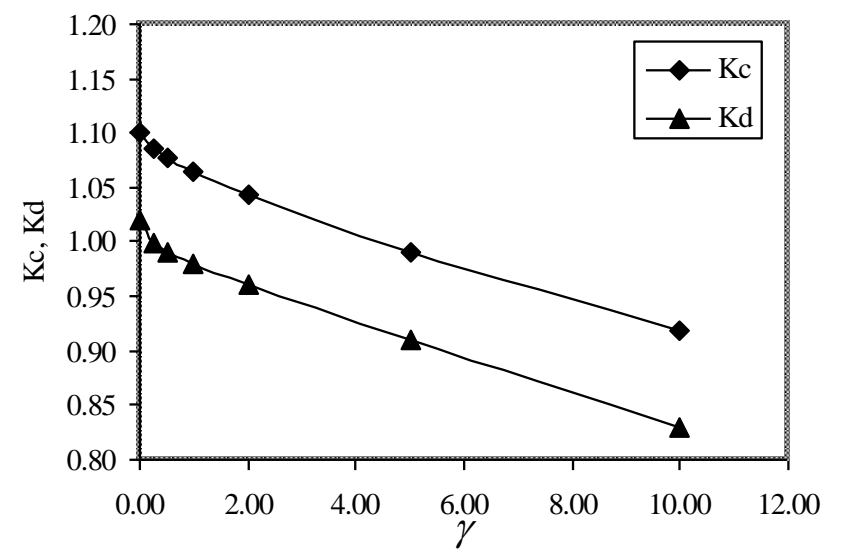

Fig. 4. Variation of normalized $K_{c}, K_{d}$ with ' $\gamma$ ' for an internal crack $(a / e=0.2, c / e=0.5, d / e=0.7$, $\left.b / e=2, \gamma=E_{2}\left(1+v_{1}\right) / E_{1}\left(1+v_{2}\right), K_{c}=k_{c} /\left(P_{0} \sqrt{(d-c) / 2}\right), K_{d}=k_{d} /\left(P_{0} \sqrt{(d-c) / 2}\right)\right)$

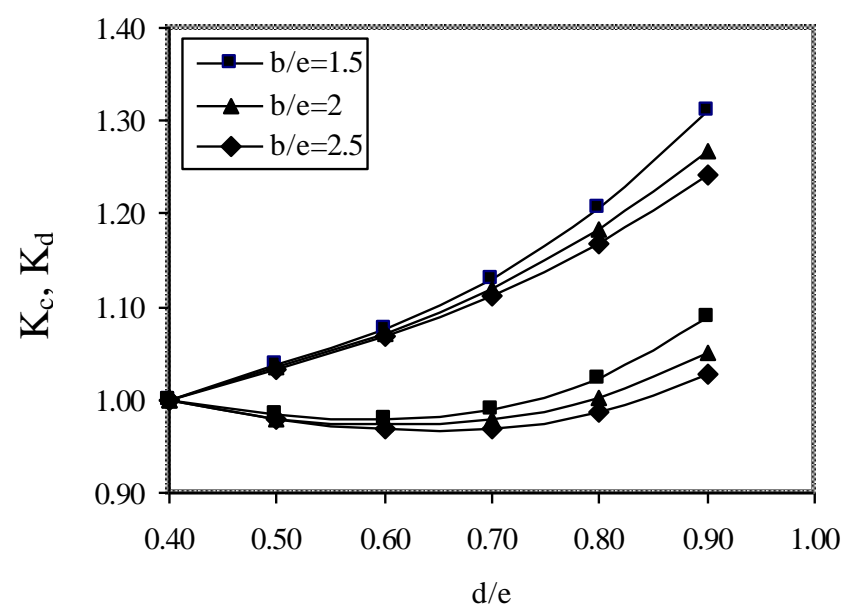

Fig. 5. Variation of normalized $K_{c}, K_{d}$ with crack length (d/e) for an internal crack $(a / e=0.1, c / e=0.4$, $\left.\gamma=0.5, K_{c}=k_{c} /\left(P_{0} \sqrt{(d-c) / 2}\right), K_{d}=k_{d} /\left(P_{0} \sqrt{(d-c) / 2}\right)\right)$ 


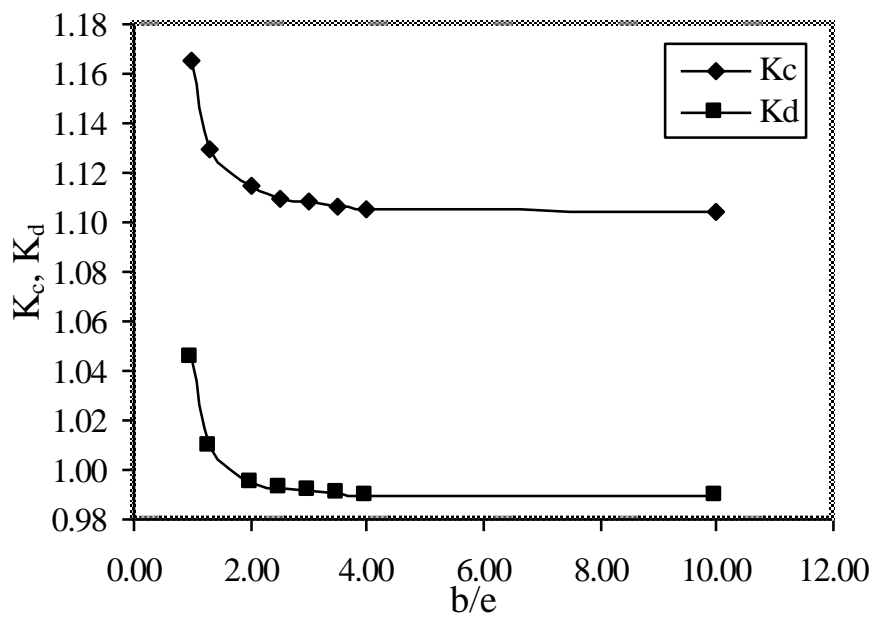

Fig. 6. Variation of normalized $K_{c}, K_{d}$ with 'b/e' for an internal crack

$$
\left(a / e=0.2, c / e=0.5, \gamma=0.5, K_{c}=k_{c} /\left(P_{0} \sqrt{(d-c) / 2}\right), K_{d}=k_{d} /\left(P_{0} \sqrt{(d-c) / 2}\right)\right)
$$

Table 1. Normalized values of $K_{c}, K_{d}$ for an internal crack

$\left(a / e=0.1, c / e=0.3, b / e=0.2, v_{1}=v_{2}=0.2\right)$
\begin{tabular}{|c|c|c|c|c|c|c|c|c|c|c|}
\hline \multirow{2}{*}{$\mathrm{d} / \mathrm{e}$} & \multicolumn{2}{|c|}{$\gamma=0$} & \multicolumn{2}{c|}{$\gamma=0.5$} & \multicolumn{2}{c|}{$\gamma=1$} & \multicolumn{2}{c|}{$\gamma=2$} & \multicolumn{2}{c|}{$\gamma=5$} \\
\cline { 2 - 12 } & $\mathrm{K}_{\mathrm{d}}$ & $\mathrm{K}_{\mathrm{c}}$ & $\mathrm{K}_{\mathrm{d}}$ & $\mathrm{K}_{\mathrm{c}}$ & $\mathrm{K}_{\mathrm{d}}$ & $\mathrm{K}_{\mathrm{c}}$ & $\mathrm{K}_{\mathrm{d}}$ & $\mathrm{K}_{\mathrm{c}}$ & $\mathrm{K}_{\mathrm{d}}$ & $\mathrm{K}_{\mathrm{c}}$ \\
\hline 0.3 & 1.0000 & 1.0000 & 1.0000 & 1.0000 & 1.0000 & 1.0000 & 1.0000 & 1.0000 & 1.0000 & 1.0000 \\
\hline 0.4 & 0.9781 & 1.0473 & 0.9759 & 1.0450 & 0.9728 & 1.0417 & 0.9748 & 1.0437 & 0.9739 & 1.0468 \\
\hline 0.5 & 0.9788 & 1.1090 & 0.9685 & 1.0976 & 0.9537 & 1.0814 & 0.9629 & 1.0914 & 0.9415 & 1.0824 \\
\hline 0.6 & 1.0008 & 1.1899 & 0.9727 & 1.1579 & 0.9325 & 1.1134 & 0.9573 & 1.1406 & 0.8864 & 1.0873 \\
\hline 0.7 & 1.0529 & 1.3017 & 0.9901 & 1.2291 & 0.9025 & 1.1333 & 0.9559 & 1.1912 & 0.7987 & 1.0544 \\
\hline 0.8 & 1.1584 & 1.4677 & 1.0275 & 1.3176 & 0.8553 & 1.1373 & 0.9584 & 1.2436 & 0.6678 & 0.9871 \\
\hline 0.9 & 1.4031 & 1.7456 & 1.1081 & 1.4358 & 0.7704 & 1.1224 & 0.9648 & 1.2988 & 0.4721 & 0.9029 \\
\hline
\end{tabular}

Table 2. Normalized values of $K_{c}, K_{d}$ for an internal crack

$\left(a / e=0.1, c / e=0.3, \gamma=0.2, v_{1}=v_{2}=0.2\right)$
\begin{tabular}{|c|c|c|c|c|c|c|c|c|c|c|}
\hline \multirow{2}{*}{$\mathrm{d} / \mathrm{e}$} & \multicolumn{2}{c}{$b / e=1.5$} & \multicolumn{2}{c|}{$b / e=2$} & \multicolumn{2}{c|}{$b / e=4$} & \multicolumn{2}{c|}{$b / e=5$} & \multicolumn{2}{c|}{$b / e=10$} \\
\cline { 2 - 12 } & $\mathrm{K}_{\mathrm{d}}$ & $\mathrm{K}_{\mathrm{c}}$ & $\mathrm{K}_{\mathrm{d}}$ & $\mathrm{K}_{\mathrm{c}}$ & $\mathrm{K}_{\mathrm{d}}$ & $\mathrm{K}_{\mathrm{c}}$ & $\mathrm{K}_{\mathrm{d}}$ & $\mathrm{K}_{\mathrm{c}}$ & $\mathrm{K}_{\mathrm{d}}$ & $\mathrm{K}_{\mathrm{c}}$ \\
\hline 0.3 & 1.0000 & 1.0000 & 1.0000 & 1.0000 & 1.0000 & 1.0000 & 1.0000 & 1.0000 & 1.0000 & 1.0000 \\
\hline 0.4 & 0.9735 & 1.0424 & 0.9728 & 1.0417 & 0.9725 & 1.0413 & 0.9725 & 1.0413 & 0.9725 & 1.0413 \\
\hline 0.5 & 0.9569 & 1.0850 & 0.9537 & 1.0814 & 0.9521 & 1.0796 & 0.9521 & 1.0795 & 0.9520 & 1.0795 \\
\hline 0.6 & 0.9406 & 1.2229 & 0.9325 & 1.1134 & 0.9285 & 1.1087 & 0.9283 & 1.1085 & 0.9282 & 1.1084 \\
\hline 0.7 & 0.9185 & 1.1526 & 0.9025 & 1.1333 & 0.8949 & 1.1239 & 0.8946 & 1.1236 & 0.8945 & 1.1234 \\
\hline 0.8 & 0.8819 & 1.1706 & 0.8553 & 1.1373 & 0.8431 & 1.1217 & 0.8427 & 1.1211 & 0.8424 & 1.1207 \\
\hline 0.9 & 0.8088 & 1.1728 & 0.7704 & 1.2224 & 0.7540 & 1.0995 & 0.7534 & 1.0986 & 0.7530 & 1.0981 \\
\hline
\end{tabular}

Figs. 7 to 9 and Tables 3 and 4 show the variation of normalized stress-intensity factors $\left(K_{d}\right)$ with increasing the crack length $(d / e)$ for the internal edge crack. As crack length decreases, the stress intensity factors at the crack tip approach a certain limit value. For a very small crack ratio $\{[(d-$ $\left.c) / e]=1 \times 10^{-6}\right\}$, the limit value is obtained as 1.12. In case the stiffness of the inner pipe is greater than that of the outer pipe $(\gamma \leq 1)$, the value of the stress intensity factor at the crack tips increase with increasing the crack length. Whereas, in case the stiffness of the inner pipe is smaller than that of the outer pipe $(\gamma>1)$, the stress intensity factor at the crack tips decreases with increasing the crack length as seen in Fig. 7.

Stress intensity factors at crack tips increase when the stiffness of the outer pipe is greater than that 
of the inner pipe. For a certain rate of shear moduli, with increasing the thickness of the outer pipe, the stress intensity factors decrease and approach a constant asymptotic value as seen in Fig. 9.

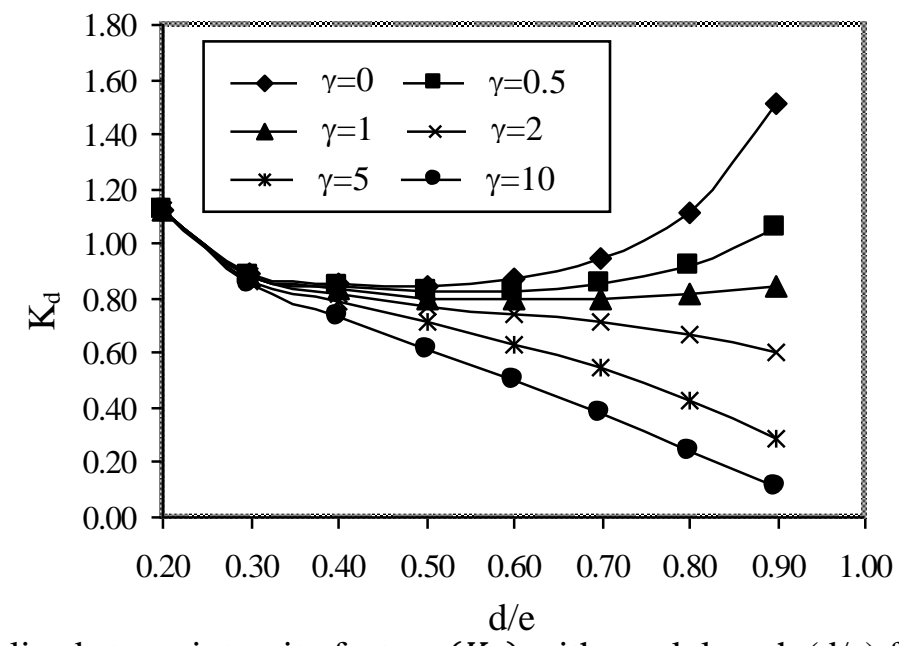

Fig. 7. Variation of normalized stress-intensity factors $\left(K_{d}\right)$ with crack length $(\mathrm{d} / \mathrm{e})$ for an internal edge crack $\left(a / e=0.2, b / e=1.5, v_{1}=v_{2}=0.2, \gamma=E_{2}\left(1+v_{1}\right) / E_{1}\left(1+v_{2}\right), K_{d}=k_{d} /\left(P_{0} \sqrt{(d-c) / 2}\right)\right)$

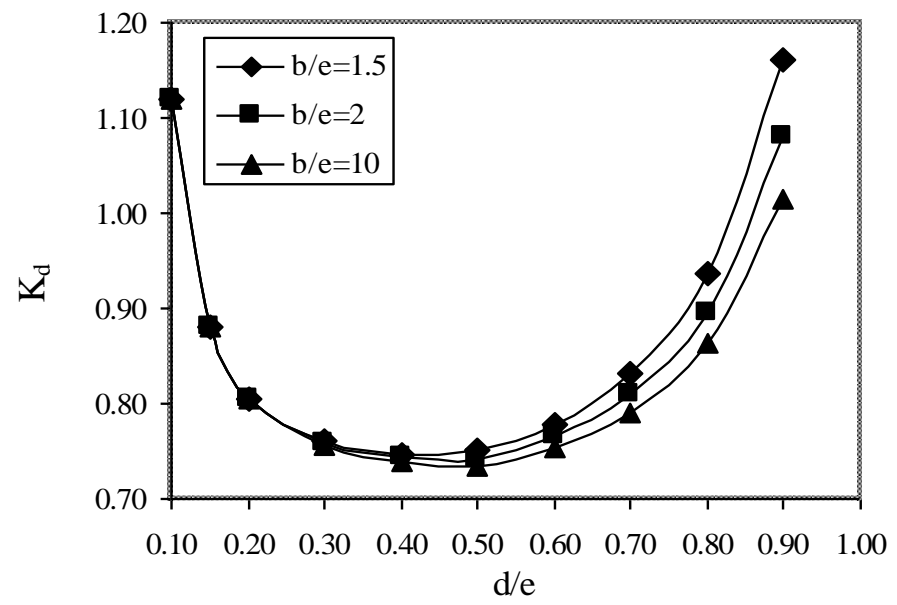

Fig. 8. Variation of normalized stress-intensity factors $\left(K_{d}\right)$ with crack length $(\mathrm{d} / \mathrm{e})$ for an internal edge crack $\left(a / e=0.1, b / e=1.5, \gamma=0.25, K_{d}=k_{d} /\left(P_{0} \sqrt{(d-c) / 2}\right)\right)$

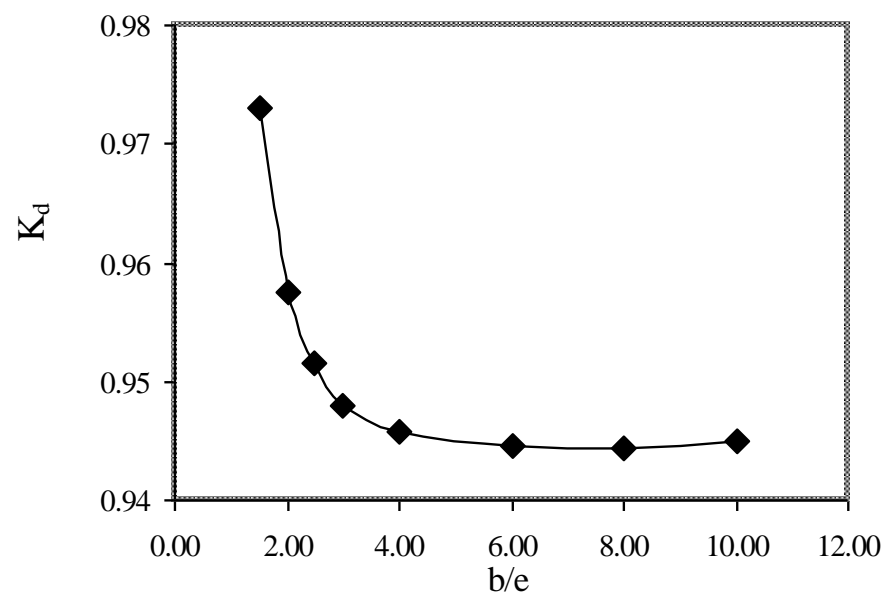

Fig. 9. Variation of normalized stress-intensity factors $\left(K_{d}\right)$ with (b/e) for an internal edge crack $\left(a / e=0.4, d / e=0.7, \gamma=0.25, v_{1}=v_{2}=0.2, K_{d}=k_{d} /\left(P_{0} \sqrt{(d-c) / 2}\right)\right)$ 
Table 3. Values of $K_{d}$ for an internal edge crack $\left(a / e=0.1, b / e=2, v_{1}=v_{2}=0.2\right)$

\begin{tabular}{|c|c|c|c|c|c|c|}
\hline \multirow{2}{*}{$\mathrm{d} / \mathrm{e}$} & $\gamma=0$ & $\gamma=0.25$ & $\gamma=0.5$ & $\gamma=1$ & $\gamma=2$ & $\gamma=10$ \\
\cline { 2 - 7 } & $\mathrm{K}_{\mathrm{d}}$ & $\mathrm{K}_{\mathrm{d}}$ & $\mathrm{K}_{\mathrm{d}}$ & $\mathrm{K}_{\mathrm{d}}$ & $\mathrm{K}_{\mathrm{d}}$ & $\mathrm{K}_{\mathrm{d}}$ \\
\hline 0.1 & 1.1215 & 1.1215 & 1.1215 & 1.1215 & 1.1215 & 1.1215 \\
\hline 0.2 & 0.8072 & 0.8066 & 0.8061 & 0.8054 & 0.8041 & 0.7945 \\
\hline 0.3 & 0.7636 & 0.7603 & 0.7582 & 0.7551 & 0.7499 & 0.7136 \\
\hline 0.4 & 0.7512 & 0.7422 & 0.7367 & 0.7287 & 0.7159 & 0.6340 \\
\hline 0.5 & 0.7637 & 0.7441 & 0.7325 & 0.7159 & 0.6902 & 0.5448 \\
\hline 0.6 & 0.8031 & 0.7644 & 0.7421 & 0.7110 & 0.6645 & 0.4439 \\
\hline 0.7 & 0.8835 & 0.8085 & 0.7672 & 0.7117 & 0.6332 & 0.3342 \\
\hline 0.8 & 1.0461 & 0.8935 & 0.8157 & 0.7170 & 0.5892 & 0.2181 \\
\hline 0.9 & 1.4442 & 1.0741 & 0.9124 & 0.7269 & 0.5182 & 0.1003 \\
\hline
\end{tabular}

Table 4. Values of $K_{d}$ for an internal edge crack $\left(a / e=0.3, \gamma=2, v_{1}=v_{2}=0.2\right)$

\begin{tabular}{|c|c|c|c|c|c|}
\hline \multirow{2}{*}{$\mathrm{d} / \mathrm{e}$} & $b / e=1.5$ & $b / e=2$ & $b / e=4$ & $b / e=5$ & $b / e=10$ \\
\cline { 2 - 6 } & $\mathrm{K}_{\mathrm{d}}$ & $\mathrm{K}_{\mathrm{d}}$ & $\mathrm{K}_{\mathrm{d}}$ & $\mathrm{K}_{\mathrm{d}}$ & $\mathrm{K}_{\mathrm{d}}$ \\
\hline 0.3 & 1.1215 & 1.1215 & 1.1215 & 1.1215 & 1.1215 \\
\hline 0.4 & 0.9077 & 0.9054 & 0.9044 & 0.9043 & 0.9043 \\
\hline 0.5 & 0.8496 & 0.8420 & 0.8385 & 0.8384 & 0.8383 \\
\hline 0.6 & 0.7968 & 0.7819 & 0.7750 & 0.7747 & 0.7746 \\
\hline 0.7 & 0.7503 & 0.7262 & 0.7152 & 0.7147 & 0.7145 \\
\hline 0.8 & 0.6980 & 0.6636 & 0.6484 & 0.6478 & 0.6475 \\
\hline 0.9 & 0.6196 & 0.5764 & 0.5576 & 0.5576 & 0.5573 \\
\hline
\end{tabular}

\section{Conclusion}

The elastostatic axisymmetric problem for long nested thick-walled cylinders with two different crack cases under uniform axial load is presented. It is determined that the stress-intensity factors vary according to the crack length, shear moduli and the rigidity of the inner and outer pipes. The values of the stress intensity factors decrease at the crack tips for the internal crack case and these values approach a constant asymptotic value. Note that, if the edge crack starts from the wall which is under tension, the stress intensity factor is always positive and decreases as the crack length increases. For a certain rate of shear moduli, with increasing the thickness of the outer pipe, the stress intensity factors decrease and approach a constant asymptotic value for the internal edge crack case.

\section{References}

Altundag Artem, H.S. and Gecit, M.R. 2002. An elastic hollow cylinder under axial tension containing a crack and two rigid inclusions of ring shape. Computers and Structures, 80, 27-30, 22772287.

Aydın, L. and Altundag Artem, H.S. 2008. Axisymmetric crack problem of thick-walled cylinder with loadings on crack surfaces.
Engineering Fracture Mechanics, 75, 6, 12941309.

Birinci, A. 2002. Axisymmetric crack problem of a thick-walled cylinder with cladding. International Journal of Engineering Science, 40, 1729-1750.

Birinci, A., Ozsahin, T.S. and Erdol, R. 2006. Axisymmetric circumferential internal crack problem of a thick-walled cylinder with inner and outer claddings. European Journal of Mechanics- A/Solids, 25, 5, 764-777.

Erdelyi, A.1953. Tables of Integral Transform, McGraw Hill, New York, USA.

Erdogan, F. and Gupta, G. D. 1972. On the numerical solution of singular integral equations. Quarterly of Applied Mathematics, 30, 533-547.

Erdogan, F. and Ratwani, M. 1972. Fracture of cylindrical and spherical shells containing a crack. Nuclear Engineering and Design, 20, 265-286.

Erdol, R. and Erdogan, F. 1978. A thick-walled cylinder with an axisymetric internal or edge crack. Journal of Applied Mechanics, 45, 281286.

Geçit, M. R. 1986. Axisymmetric contact problem for a semi-infinite cylinder and a half space. International Journal of Engineering Science, 24(8), 1245-1256. 
Gupta, G. D. 1974. The analysis of the semi-infinite cylinder problem. International Journal of Solids and Structures, 10, 137-148.

Gupta, G. D. and Erdogan, F. 1974. The problem of edge cracks in an infinite strip. Journal of Applied Mechanics, 41, 1001-1006.

Krenk, S. 1975. A note on the use of the interpolation polynomial for solutions of singular integral equations. Quarterly of Applied Mathematics, $32,479-485$.

Muskhelishvili, N.I. 1958. Singular Integral Equations, Noordhoff Publishing, Leiden, Netherland.

Nied, H. F. and Erdogan, F. 1983. The elasticity problem for a thick-walled cylinder containing a circumferential crack. International Journal of Fracture, 22, 277-301.

Sneddon, L. N. and Welch, J. Y. 1963. A note on the distribution of stress in a cylinder containing a penny-shaped crack. . International Journal of Engineering Science, 1, 411-419.

Sneddon, L.N. and Tait, R. J. 1963. The effect of a penny shaped crack on distribution of stress in a long circular cylinder. International Journal of Engineering Science, 1, 391-409.

Toygar, M.E. and Gecit, M.R. 2006. Crack infinite cylinder with two rigid inclusions under axisymmetric tension. International Journal of Solids and Structures, 43, 16, 4777-4794.

\section{APPENDIX A}

$$
\begin{aligned}
& \gamma=\frac{E_{2}\left(1+v_{1}\right)}{E_{1}\left(1+v_{2}\right)} \\
& m^{*}=-\frac{1}{\xi^{2}} \sqrt{\frac{2}{\pi} \frac{E_{1}}{2\left(1-v_{1}^{2}\right)}} \\
& z_{1}=m^{*} \int_{c}^{d} \rho G(\rho)\left\{\xi^{2} a \rho I_{0}(\xi a) K_{0}(\xi \rho)-\left[2\left(1-v_{1}\right)+\xi^{2} a^{2}\right] I_{1}(\xi a) K_{1}(\xi \rho)\right. \\
& \left.+\xi a I_{0} K_{1}(\xi \rho)-\xi \rho I_{1}(\xi a) K_{0}(\xi \rho)\right\} d \rho \\
& z_{2}=m^{*} \int_{c}^{d} \rho G(\rho)\left\{\xi a I_{0}(\xi a) K_{1}(\xi \rho)-\xi \rho I_{1}(\xi a) K_{0}(\xi \rho)\right\} d \rho \\
& z_{3}=m^{*} \int_{c}^{d} \rho G(\rho)\left\{2\left(1-v_{1}\right) K_{1}(\xi e) I_{1}(\xi \rho)+\xi e K_{0}(\xi e) I_{1}(\xi \rho)-\xi \rho K_{1}(\xi e) I_{0}(\xi \rho)\right\} d \rho \\
& z_{4}=m^{*} \int_{c}^{d} \rho G(\rho)\left\{2\left(1-v_{1}\right) K_{0}(\xi e) I_{1}(\xi \rho)-\xi e K_{0}(\xi e) I_{0}(\xi \rho)+\xi \rho K_{1}(\xi e) I_{1}(\xi \rho)\right\} d \rho \\
& z_{5}=m^{*} \int_{c}^{d} \rho G(\rho)\left\{\xi^{2} e \rho K_{0}(\xi e) I_{0}(\xi \rho)-\left[2\left(1-v_{1}\right)+\xi^{2} e^{2}\right] K_{1}(\xi e) I_{1}(\xi \rho)-\xi e K_{0}(\xi e) I_{1}(\xi \rho)\right. \\
& \left.+\xi \rho K_{1}(\xi e) I_{0}(\xi \rho)\right\} d \rho \\
& z_{6}=m^{*} \int_{c}^{d} \rho G(\rho)\left\{-\xi e K_{0}(\xi e) I_{1}(\xi \rho)+\xi \rho K_{1}(\xi e) I_{0}(\xi \rho)\right\} d \rho \\
& a_{i}=\left\langle\left\{2\left(1-v_{1}\right)\left[K_{1}(\xi a) I_{1}(\xi a)+\xi a y_{1}\right]+(\xi a)^{2} y_{2}\right\} c_{i}+\xi a y_{1}\left\{(\xi a)^{2}-2\left(1-v_{1}\right)\left(1-2 v_{1}\right)\right\} d_{i}\right. \\
& \left.+\left\{2\left(1-v_{1}\right) I_{1}(\xi a)+\xi a I_{0}(\xi a)\right\} \delta_{i 1} \Delta+\left\{\left(1-2 v_{1}\right) \xi a I_{0}(\xi a)+(\xi a)^{2} I_{1}(\xi a)\right\} \delta_{i 2} \Delta\right\rangle \\
& / y_{7} \\
& b_{i}=\left\langle-\xi a y_{1} c_{i}-\left\{2\left(1-v_{1}\right)\left[K_{1}(\xi a) I_{1}(\xi a)-\xi a y_{1}\right]+(\xi a)^{2} y_{2}\right\} d_{i}-I_{1}(\xi a) \delta_{i 1} \Delta\right. \\
& \left.-\left[I_{1}(\xi a)-(\xi a) I_{o}(\xi a)\right] \delta_{i 2} \Delta\right\rangle / y_{7} \\
& c_{i}=\left\{m^{2} \alpha_{1}\left(-x_{31} y_{i 1}+x_{42} y_{i 2}\right)-m\left(\alpha_{17} y_{i 1}-\alpha_{18} y_{i 2}+\alpha_{19} y_{i 3}-\alpha_{20} y_{i 4}\right)\right. \\
& \left.+\alpha_{6}\left(-x_{12} y_{i 3}+x_{22} y_{i 4}\right)\right\}\left(\delta_{i 1}+\delta_{i 2}\right) \\
& -y_{7}\left\{\left(m \alpha_{20}+x_{22} \alpha_{6}\right) \delta_{i 3}-\left(m \alpha_{19}+x_{12} \alpha_{6}\right) \delta_{i 4}+m\left(m x_{42} \alpha_{1}+\alpha_{18}\right) \delta_{i 5}\right. \\
& \left.-m\left(m x_{32} \alpha_{1}+\alpha_{17}\right) \delta_{i 6}\right\}
\end{aligned}
$$




$$
\begin{aligned}
& d_{i}=\left\{m^{2} \alpha_{1}\left(x_{31} y_{i 1}-x_{41} y_{i 2}\right)+m\left(\alpha_{13} y_{i 1}-\alpha_{14} y_{i 2}+\alpha_{15} y_{i 3}-\alpha_{16} y_{i 4}\right)\right. \\
& \left.+\alpha_{6}\left(x_{11} y_{i 3}-x_{21} y_{i 4}\right)\right\}\left(\delta_{i 1}+\delta_{i 2}\right) \\
& -\left\{y_{7}\left(m \alpha_{16}+x_{21} \alpha_{6}\right) \delta_{i 3}-\left(m \alpha_{15}+x_{1} \alpha_{6}\right) \delta_{i 4}+m\left(m x_{41} \alpha_{1}+\alpha_{14}\right) \delta_{i 5}\right. \\
& \left.-m\left(m x_{31} \alpha_{1}+\alpha_{13}\right) \delta_{i 6}\right\} \quad(i=1, \ldots, 6) \\
& \Delta=-m^{2} \alpha_{11} \alpha_{1}+m\left(-\alpha_{5} \alpha_{8}+\alpha_{3} \alpha_{10}+\alpha_{4} \alpha_{7}-\alpha_{9} \alpha_{2}\right)-\alpha_{6} \alpha_{12} \\
& y_{1}=I_{0}(\xi a) K_{1}(\xi a)+I_{1}(\xi a) K_{0}(\xi a) \\
& y_{2}=I_{0}(\xi a) K_{0}(\xi a)+I_{1}(\xi a) K_{1}(\xi a) \\
& y_{3}=K_{0}(\xi b) I_{1}(\xi b)+I_{0}(\xi b) K_{1}(\xi b) \\
& y_{4}=I_{0}(\xi b) K_{0}(\xi b)+I_{1}(\xi b) K_{1}(\xi b) \\
& y_{5}=I_{1}(\xi e)-\xi e I_{0}(\xi e) \\
& y_{6}=K_{1}\left(\xi_{e}\right)+\xi_{e} K_{0}\left(\xi_{e}\right) \\
& y_{7}=(\xi a)^{2}\left[I_{1}(\xi a)^{2}-I_{0}(\xi a)^{2}\right]+2\left(1-v_{1}\right) I_{1}(\xi a)^{2} \\
& y_{8}=(\xi b)^{2}\left[I_{1}(\xi b)^{2}-I_{0}(\xi b)^{2}\right]+2\left(1-v_{2}\right) I_{1}(\xi b)^{2} \\
& y_{11}=I_{1}(\xi e)\left\{2\left(1-v_{1}\right) I_{1}(\xi a)+\xi a I_{0}(\xi a)\right\}-I_{1}(\xi a)\left\{2\left(1-v_{1}\right) I_{1}(\xi e)+\xi e I_{0}(\xi e)\right\} \\
& y_{12}=y_{5}\left\{2\left(1-v_{1}\right) I_{1}(\xi a)+\xi a I_{0}(\xi a)\right\}-I_{1}(\xi a)\left\{2\left(v_{1}-1\right) \xi e I_{0}(\xi e)-(\xi e)^{2} I_{1}(\xi e)\right\} \\
& y_{13}=I_{0}(\xi e)\left\{2\left(1-v_{1}\right) I_{1}(\xi a)+\xi a I_{0}(\xi a)\right\}-I_{1}(\xi a)\left\{4\left(1-v_{1}\right) I_{0}(\xi e)+\xi e I_{1}(\xi e)\right\} \\
& y_{14}=\xi e I_{0}(\xi e) I_{1}(\xi a)-I_{1}(\xi e)\left\{2\left(1-v_{1}\right) I_{1}(\xi a)+\xi a I_{0}(\xi a)\right\} \\
& y_{21}=I_{1}(\xi e)\left\{\left(1-2 v_{1}\right) \xi a I_{0}(\xi a)+(\xi a)^{2} I_{1}(\xi a)\right\} \\
& +\left[I_{1}(\xi a)-(\xi a) I_{0}(\xi a)\right]\left\{2\left(1-v_{1}\right) I_{1}(\xi e)+\xi e I_{0}(\xi e)\right\} \\
& y_{22}=y_{5}\left\{\left(1-2 v_{1}\right) \xi a I_{0}(\xi a)+(\xi a)^{2} I_{1}(\xi a)\right\} \\
& +\left[I_{1}(\xi a)-(\xi a) I_{0}(\xi a)\right]\left\{\left(2 v_{1}-1\right) \xi e I_{0}(\xi e)-(\xi e)^{2} I_{1}(\xi e)\right\} \\
& y_{23}=I_{0}(\xi e)\left\{\left(1-2 v_{1}\right) \xi a I_{0}(\xi a)+(\xi a)^{2} I_{1}(\xi a)\right\} \\
& +\left[I_{1}(\xi a)-(\xi a) I_{0}(\xi a)\right]\left\{4\left(1-v_{1}\right) I_{0}(\xi e)+\xi e I_{1}(\xi e)\right\} \\
& y_{24}=\xi e\left[I_{1}(\xi a)-(\xi a) I_{0}(\xi a)\right] I_{0}(\xi e)+I_{1}(\xi e)\left\{\left(1-2 v_{1}\right) \xi a I_{0}(\xi a)+(\xi a)^{2} I_{1}(\xi a)\right\} \\
& x_{11}=-I_{1}(\xi e)\left\{2\left(1-v_{1}\right)\left[K_{1}(\xi a) I_{1}(\xi a)+\xi a y_{1}\right]+(\xi a)^{2} y_{2}\right\}+\xi^{2} e a y_{1} I_{0}(\xi e)+y_{7} K_{1}(\xi e) \\
& x_{12}=-\xi a y_{1} I_{1}(\xi e)\left\{(\xi a)^{2}-2\left(1-v_{1}\right)\left(1-2 v_{1}\right)\right\} \\
& +\xi e I_{0}(\xi e)\left\{2\left(1-v_{1}\right)\left[K_{1}(\xi a) I_{1}(\xi a)-\xi a y_{1}\right]+(\xi a)^{2} y_{2}\right\} \\
& x_{13}=I_{1}(\xi e)\left\{2\left(1-v_{1}\right)\left[K_{1}(\xi b) I_{1}(\xi b)+\xi b y_{3}\right]+(\xi b)^{2} y_{4}\right\}-\xi^{2} e b y_{3} I_{0}(\xi e)-y_{7} K_{1}(\xi e) \\
& x_{14}=-\xi b y_{3} I_{1}(\xi e)\left\{(\xi b)^{2}-2\left(1-v_{1}\right)\left(1-2 v_{2}\right)\right\} \\
& -\xi e I_{0}(\xi e)\left\{2\left(1-v_{2}\right)\left[K_{1}(\xi b) I_{1}(\xi b)-\xi b y_{3}\right]+(\xi b)^{2} y_{4}\right\} \\
& x_{21}=I_{0}(\xi e)\left\{2\left(1-v_{1}\right)\left[K_{1}(\xi a) I_{1}(\xi a)+\xi a y_{1}\right]+(\xi a)^{2} y_{2}\right\}-\xi^{2} a y_{1} I_{0}(\xi e)-y_{7} K_{1}(\xi e) \\
& x_{22}=\left\{2\left(1-v_{1}\right)\left[K_{1}(\xi a) I_{1}(\xi a)-\xi a y_{1}\right]+(\xi a)^{2} y_{2}\right\}\left\{4\left(1-v_{1}\right) I_{0}(\xi e)+\xi e I_{1}(\xi e)\right\} \\
& +\xi a y_{1} I_{0}(\xi e)\left\{(\xi a)^{2}-2\left(1-v_{1}\right)\left(1-2 v_{1}\right)\right\} \\
& +K_{1}(\xi e)\left\{-4\left(1-v_{1}\right) K_{0}(\xi e)+\xi e K_{1}(\xi e)\right\} \\
& x_{23}=-I_{0}(\xi e)\left\{2\left(1-v_{1}\right)\left[K_{1}(\xi b) I_{1}(\xi b)+\xi a y_{3}\right]+(\xi b)^{2} y_{4}\right\} \\
& +\xi b y_{3}\left\{4\left(1-v_{2}\right) I_{0}(\xi e)+\xi e I_{1}(\xi e)\right\}-y_{8} K_{0}(\xi e) \\
& x_{24}=-\xi b y_{3} I_{0}(\xi e)\left\{(\xi b)^{2}-2\left(1-v_{2}\right)\left(1-2 v_{2}\right)\right\}+y_{8}\left\{4\left(1-v_{2}\right) K_{0}(\xi e)-\xi e K_{1}(\xi e)\right\} \\
& +\left\{2\left(1-v_{2}\right)\left[K_{1}(\xi b) I_{1}(\xi b)-\xi b y_{3}\right]+(\xi b)^{2} y_{4}\right\}\left\{4\left(1-v_{2}\right) I_{0}(\xi e)+\xi e I_{1}(\xi e)\right\} \\
& x_{31}=y_{5}\left\{2\left(1-v_{1}\right)\left[K_{1}(\xi a) I_{1}(\xi a)+\xi a y_{1}\right]+(\xi a)^{2} y_{2}\right\}-\xi^{2} e a y_{1}\left\{\left(2 v_{1}-1\right) I_{0}(\xi e)-\xi e I_{1}(\xi e)\right\} \\
& -y_{6} y_{7} \\
& x_{32}=\xi a y_{1} y_{5}\left\{(\xi a)^{2}-2\left(1-v_{1}\right)\left(1-2 v_{1}\right)\right\}+y_{7}\left\{\left(1-2 v_{1}\right) \xi_{e} K_{0}\left(\xi_{e}\right)-\left(\xi_{e}\right)^{2} K_{1}\left(\xi_{e}\right)\right\} \\
& -\left\{\left(2 v_{1}-1\right) \xi e I_{0}(\xi e)-(\xi e)^{2} I_{1}(\xi e)\right\}\left\{2\left(1-v_{1}\right)\left[K_{1}(\xi a) I_{1}(\xi a)-\xi a y_{1}\right]\right. \\
& \left.+(\xi a)^{2} y_{2}\right\} \\
& x_{33}=y_{6} y_{8}-y_{5}\left\{2\left(1-v_{2}\right)\left[K_{1}(\xi b) I_{1}(\xi b)+\xi b y_{3}\right]+(\xi b)^{2} y_{4}\right\} \\
& -\xi^{2} e b y_{3}\left\{\left(1-2 v_{2}\right) I_{0}(\xi e)+\xi e I_{1}(\xi e)\right\} \\
& x_{34}=-\xi b y_{3} y_{5}\left\{(\xi b)^{2}-2\left(1-v_{2}\right)\left(1-2 v_{2}\right)\right\}+y_{8}\left\{\left(2 v_{2}-1\right) \xi e K_{0}(\xi e)+(\xi e)^{2} K_{1}(\xi e)\right\} \\
& -\left\{2\left(1-v_{2}\right)\left[K_{1}(\xi b) I_{1}(\xi b)-\xi b y_{3}\right]+(\xi b)^{2} y_{4}\right\}\left\{\left(1-2 v_{2}\right) \xi e I_{0}(\xi e)\right. \\
& \left.+(\xi e)^{2} I_{1}(\xi e)\right\} \\
& x_{41}=I_{1}(\xi e)\left\{2\left(1-v_{1}\right)\left[K_{1}(\xi a) I_{1}(\xi a)+\xi a y_{1}\right]+(\xi a)^{2} y_{2}\right\}-\xi a\left\{2\left(1-v_{1}\right) I_{1}(\xi e)+(\xi e) I_{0}(\xi e)\right\} \\
& -y_{7} K_{1}(\xi e)
\end{aligned}
$$




$$
\begin{aligned}
& x_{42}=\xi a y_{1} I_{1}(\xi e)\left\{(\xi a)^{2}-2\left(1-v_{1}\right)\left(1-2 v_{1}\right)\right\}+y_{7}\left\{2\left(1-v_{1}\right) K_{1}(\xi e)-\xi e K_{0}(\xi e)\right\} \\
& -\left\{2\left(1-v_{1}\right)\left[K_{1}(\xi a) I_{1}(\xi a)-\xi a y_{1}\right]+(\xi a)^{2} y_{2}\right\}\left\{2\left(1-v_{1}\right) I_{1}(\xi e)+(\xi e) I_{1}(\xi e)\right\} \\
& x_{43}=-I_{1}(\xi e)\left\{2\left(1-v_{1}\right)\left[K_{1}(\xi b) I_{1}(\xi b)+\xi b y_{3}\right]+(\xi b)^{2} y_{4}\right\} \\
& +\xi b y_{3}\left\{2\left(1-v_{2}\right) I_{1}(\xi e)+(\xi e) I_{0}(\xi e)\right\}+y_{8} K_{1}(\xi e) \\
& x_{44}=-\xi b y_{3} I_{1}(\xi e)\left\{(\xi b)^{2}-2\left(1-v_{1}\right)\left(1-2 v_{2}\right)\right\}+y_{8}\left\{-2\left(1-v_{2}\right) K_{1}\left(\xi_{e}\right)+\xi_{e} K_{0}(\xi e)\right\} \\
& +\left\{2\left(1-v_{2}\right)\left[K_{1}(\xi b) I_{1}(\xi b)-\xi b y_{3}\right]+(\xi b)^{2} y_{4}\right\}\left\{2\left(1-v_{2}\right) I_{1}(\xi e)+(\xi e) I_{0}(\xi e)\right\} \\
& \alpha_{1}=x_{13} x_{24}-x_{23} x_{14} \quad \alpha_{2}=x_{14} x_{22}-x_{12} x_{24} \quad \alpha_{3}=x_{12} x_{23}-x_{13} x_{22} \quad \alpha_{4}=x_{32} x_{43}-x_{42} x_{33} \\
& \alpha_{5}=x_{34} x_{42}-x_{44} x_{32} \quad \alpha_{6}=x_{33} x_{44}-x_{34} x_{43} \quad \alpha_{7}=x_{21} x_{14}-x_{11} x_{24} \quad \alpha_{8}=x_{11} x_{23}-x_{13} x_{21} \\
& \alpha_{9}=x_{31} x_{43}-x_{41} x_{33} \alpha_{10}=x_{41} x_{34}-x_{31} x_{44} \alpha_{11}=x_{42} x_{31}-x_{41} x_{32} \alpha_{12}=x_{11} x_{22}-x_{21} x_{12} \\
& \alpha_{13}=x_{33} \alpha_{7}+x_{34} \alpha_{8} \quad \alpha_{14}=x_{43} \alpha_{7}+x_{44} \alpha_{8} \quad \alpha_{15}=x_{14} \alpha_{9}+x_{13} \alpha_{10} \quad \alpha_{16}=x_{24} \alpha_{9}+x_{23} \alpha_{10} \\
& \alpha_{17}=x_{33} \alpha_{2}+x_{34} \alpha_{3} \quad \alpha_{18}=x_{43} \alpha_{2}+x_{44} \alpha_{3} \quad \alpha_{19}=x_{14} \alpha_{4}+x_{13} \alpha_{5} \quad \alpha_{20}=x_{14} \alpha_{4}+x_{23} \alpha_{5}
\end{aligned}
$$

